Proc. HCIS-X - Berlin, 1997 (phys. stat. sol.(b), to be published)

\title{
Coulomb-correlation effects on the non-linear optical properties of realistic quantum wires
}

\author{
Fausto Rossi* and Elisa Molinari \\ Istituto Nazionale Fisica della Materia (INFM) and \\ Dipartimento di Fisica, Università di Modena \\ via Campi 213/A, I-41100 Modena, Italy
}

(March 21, 2018)

\begin{abstract}
We review recent results on the linear and non-linear optical response of realistic quantum-wire structures. Our theoretical approach is based on a set of generalized semiconductor Bloch equations, and allows a full threedimensional multisubband description of Coulomb correlation for any shape of the confinement profile, thus permitting a direct comparison with experiments for available state-of-the-art wire structures.

Our results show that electron-hole Coulomb correlation removes the onedimensional band-edge singularities from the absorption spectra, whose shape results to be heavily modified with respect to the ideal free-particle case over the whole range of photoexcited carrier densities.
\end{abstract}

Classification scheme: 78.66.F, 73.20.D

Typeset using REVTEX

*phone: +39 (59) 586072 — fax: +39 (59) 367488 - E-mail: Rossi@UniMo.It 


\section{INTRODUCTION}

The dominant role played by Coulomb correlation in the optical response of semiconductors and its dependence on dimensionality has now been long recognized [1]. More recently, increasing interest has been devoted to one-dimensional (1D) structures [2], prompted by promising advances in quantum-wire fabrication and application, e.g. in quantum-wire lasers. The main goal of such effort in basic research as well as technological applications is to achieve structures with improved optical efficiency as compared to two-dimensional (2D) and three-dimensional (3D) ones.

A common argument in favour of this effort is based on the well known van Hove divergence in the $1 \mathrm{D}$ joint density-of-states (DOS), which is expected to give rise to very sharp peaks in the optical spectra of $1 \mathrm{D}$ structures. Such prediction is however based on free-particle properties of ideal 1D systems and it ignores any disorder-induced and Coulombcorrelation effects.

As pointed out in the pioneering papers by Ogawa et al. [3], electron-hole correlation is expected to strongly influence the optical spectra of 1D systems. Their theoretical investigation - based on a single-subband-model solution of the 1D Schrödinger equation in terms of a modified 1D Coulomb potential [3] - shows that the inverse-square-root singularity in the 1D DOS at the band edge is smoothed when electron-hole correlation is taken into account. The question is whether one can expect that the above theoretical predictions, obtained for model 1D systems, also apply to the real quantum wires made available by the present technology. Indeed, wires with the best optical quality presently include structures obtained by epitaxial growth on non-planar substrates (V-shaped wires) [2, 4, 5], or by cleaved-edge quantum well overgrowth (T-shaped wires) [6,7]. Owing to the shape of the confinement potential, these systems are far from an ideal 1D character. While quasi-1D confinement has been demonstrated for the lowest level [2,40], excited states gradually approach a 2Dlike behaviour. Moreover, in the available samples the subband separation is still relatively small, so that the coupling between different subbands may be important. 
From an experimental point of view, it is a matter of fact that, while $2 \mathrm{D}$ features are clearly observed in photoluminescence excitation spectra of quantum wells, so far no "sharp" 1D features have been detected in the corresponding spectra of quantum wires. This is true despite the high quality of some of these structures, whose 1D character has been independently established by other methods [2, [4 6]. However, the measured spectra are expected to be also strongly influenced by disorder-induced inhomogeneous broadening [8] and, therefore, it has been sofar difficult to identify the role played by electron-hole correlation.

From all these considerations, the following questions need to be answered:

Are electron-hole correlation effects playing a dominant role also in realistic quantumwire structures? If so, are these expected to hinder the possible advantages of the reduced dimensionality for relevant values of temperature and carrier density?

\section{THEORETICAL APPROACH}

To answer these questions, a full 3D approach for the analysis of Coulomb correlation in realistic quantum wires has been recently proposed [9,10]. This theoretical scheme, described in [9], is based on a generalization of the well-known semiconductor Bloch equations (SBE) [1] to the case of a multisubband wire.

More specifically, by denoting with $\left\{k_{z} \nu^{e / h}\right\}$ the free electron and hole states $\left(k_{z}\right.$ and $\nu^{e / h}$ being, respectively, the wavevector along the wire direction, $z$, and the subband index corresponding to the confinement potential in the $x y$ plane), we consider as kinetic variables

the various distribution functions of electrons and holes $f_{k_{z} \nu}^{e / h}$ as well as the corresponding diagonal $\left(\nu^{e}=\nu^{h}=\nu\right)$ interband polarizations $p_{k_{z} \nu}$. This kinetic description is a generalization to 1D systems of a standard approach for the study of bulk semiconductors [1] recently applied also to superlattice structures [11]. Within our $k_{z} \nu$ representation, the SBE, describing the time evolution of the above kinetic variables, are written as

$$
\frac{\partial}{\partial t} f_{ \pm k_{z} \nu}^{e / h}=\frac{1}{i \hbar}\left(\mathcal{U}_{k_{z} \nu} p_{k_{z} \nu}^{*}-\mathcal{U}_{k_{z} \nu}^{*} p_{k_{z} \nu}\right)+\left.\frac{\partial}{\partial t} f_{ \pm k_{z} \nu}^{e / h}\right|_{i n c o}
$$




$$
\frac{\partial}{\partial t} p_{k_{z} \nu}=\frac{1}{i \hbar}\left(\mathcal{E}_{k_{z} \nu}^{e}+\mathcal{E}_{-k_{z} \nu}^{h}\right) p_{k_{z} \nu}+\frac{1}{i \hbar} \mathcal{U}_{k_{z} \nu}\left(1-f_{k_{z} \nu}^{e}-f_{-k_{z} \nu}^{h}\right)+\left.\frac{\partial}{\partial t} p_{k_{z} \nu}\right|_{\text {inco }},
$$

where $\mathcal{U}_{k_{z} \nu}$ and $\mathcal{E}_{k_{z} \nu}^{e / h}$ are, respectively, the renormalized fields and subbands, whose explicit form involves the full 3D Coulomb potential [9]. The \pm sign in Eq. (11) refers to electrons $(e)$ and holes $(h)$, respectively, while the last terms on the rhs of Eq. (1) denote the contributions due to incoherent processes, e.g. carrier-carrier and carrier-phonon scattering.

In this paper we focus on the quasi-equilibrium regime. Therefore, Fermi-Dirac $f_{k_{z} \nu}^{e / h}$ are assumed and the solution of the set of SBE (1) simply reduces to the solution of the polarization equation. This is performed by means of a direct numerical evaluation of the stationary solutions, i.e. polarization eigenvalues and eigenvectors. These two ingredients fully determine the absorption spectrum as well as the exciton wavefunction in 3D real space. In particular, the electron-hole correlation function vs. the relative free coordinate $z=z^{e}-z^{h}$ is given by: $g(z) \propto \sum_{k_{z} k_{z}^{\prime} \nu} p_{k_{z} \nu}^{*} p_{k_{z}^{\prime} \nu} e^{i\left(k_{z}^{\prime}-k_{z}\right) z}$.

The main ingredients entering our calculation are then the single-particle energies and wavefunctions, which in turn are numerically computed starting from the real shape of the 2D confinement potential deduced from TEM, as in Ref. [5].

\section{NUMERICAL RESULTS}

The above theoretical scheme has been applied to realistic V-and T-shaped wire structures. In particular, here we show results for the GaAs/AlGaAs V-wires of Ref. [5] and the GaAs/AlAs T-shaped wire of Ref. [7] (sample S2 $\left[d_{1}=d_{2}=53 \AA\right]$ ).

In order to better illustrate the role played by electron-hole correlation, in Fig. 1 we first show the linear-absorption spectra obtained when taking into account the lowest wire subband only. Here, results of our Coulomb-correlated (CC) approach are compared with those of the free-carrier (FC) model [12]. For both V-shaped [Fig. 1(a)] and T-shaped [Fig. 1(b)] structures, electron-hole correlation introduces two important effects: First, the excitonic peak arises below the onset of the continuum, with different values of binding energies (about 12 and $16 \mathrm{meV}$, respectively) in good agreement with experiments [5]. As 
discussed in [10], this difference is mainly ascribed to the different barrier height while the excitonic confinement is found to be shape (V vs. T) independent. Second, we find a strong suppression of the 1D DOS singularity, in agreement with previous investigations based on simplified 1D models [3].

Let us now discuss the physical origin of the dramatic suppression of the band-edge singularity in the CC absorption spectrum [solid lines in Fig. 1]. Since the optical absorption is proportional to the product of the electron-hole DOS and the oscillator strength (OS), we have studied these two quantities separately. Figure 2(a) shows that the quantity which is mainly modified by CC is the OS. Here, the ratio between the CC and FC OS is plotted as a function of the excess energy with respect to the band edge (solid line). This ratio is always less than one and, in agreement with the results of $1 \mathrm{D}$ models [3], it goes to zero at the band edge. Such vanishing behaviour is found to dominate the 1D DOS singularity (dashed line) and, as a result, the absorption spectrum exhibits a regular behaviour at the band edge [solid lines in Fig. 1].

Since the OS reflects the value of the correlation function $g(z)$ for $z=0$ [3], i.e. the probability of finding electron and hole at the same place, the vanishing behaviour of the OS in Fig. 2(a) seems to indicate a sort of electron-hole "effective repulsion". This is confirmed by a detailed analysis of the electron-hole correlation function, $g(z)$, reported in Fig. 2(b). Here $g(z)$ (corresponding to the square of the exciton wavefunction in a 1D model) is plotted for three different values of the excess energy. We clearly see that the values of $g$ for $z=0$ correspond to the values of the OS ratio at the same energies [solid line in Fig. 2(a)]. Moreover, we notice the presence of a true "electron-hole correlation hole", whose spatial extension strongly increases when approaching the band edge.

The above analysis provides a positive answer to our first question: also for realistic quantum-wire structures electron-hole correlation leads to a strong suppression of the 1D band-edge singularity in the linear-absorption spectrum. Contrary to the 2D and 3D case, the Sommerfeld factor, i.e. the ratio between the $\mathrm{CC}$ and FC absorption, results to be less than unity. 
Finally, in order to answer our second and more crucial question, we must consider that most of the potential quantum-wire applications, i.e. 1D lasers and modulators, operate in strongly non-linear-response regimes [2]. In such conditions, the above linear-response analysis has to be generalized taking into account additional factors as: (i) screening effects, (ii) band renormalization, (iii) space-phase filling. We want to stress that all these effects are already accounted for in our SBE (1) [9].

Figure 3 shows the first quantitative analysis of non-linear absorption spectra of realistic V-shaped wire structures for different carrier densities at room temperature. In Fig. 3(a) we show as a reference the results obtained by including the lowest subband only. In the low-density limit (case A: $n=10^{4} \mathrm{~cm}^{-1}$ ) we clearly recognize the exciton peak. With increasing carrier density, the strength of the excitonic absorption decreases due to phasespace filling and screening of the attractive electron-hole interaction, and moreover the band renormalization leads to a red-shift of the continuum. Above the Mott density (here about $8 * 10^{5} \mathrm{~cm}^{-1}$ ), the exciton completely disappears. At a density of $4 * 10^{6} \mathrm{~cm}^{-1}$ (case D) the spectrum already exhibits a negative region corresponding to stimulated emission, i.e. gain regime. As desired, the well pronounced gain spectrum extends over a limited energy region (smaller than the thermal energy); However, its shape results to differ considerably from the ideal FC one. The FC result is plotted in the same figure and marked with diamonds; note that it has been shifted in energy to align the onset of the absorption, to allow a better comparison of the line-shapes [13]. The typical shape of the band-edge singularity in the ideal FC gain spectrum results to be strongly modified by electron-hole correlation. Also at this relatively high carrier density the OS corresponding to the $\mathrm{CC}$ model goes to zero at the band edge as for the low-density limit [Fig. 2(a)]. As a consequence, the FC peak is strongly suppressed and only its high-energy part survives. The overall effect is a broader and less pronounced gain region.

Finally, Fig. 3(b) shows the non-linear spectra corresponding to the realistic case of a 12-subband V-shaped wire. In comparison with the single-subband case [Fig3(a)], the multisubband nature is found to play an important role in modifying the typical shape of 
the gain spectra, which for both $\mathrm{CC}$ and FC models result to extend over a range much larger than that of the single-subband case for the present wire geometry [Fig. 3(a)]. In addition, the Coulomb-induced suppression of the single-subband singularities, here also due to intersubband-coupling effects, tends to further reduce the residual structures in the gain profile. Thus, even in the ideal case of a quantum wire with negligible disorder- and scattering-induced broadening, our analysis indicates that, for the typical structure considered, the shape of the absorption spectra over the whole density range differs considerably from the relatively sharp FC spectrum of Fig. 1.

\section{CONCLUSIONS}

We have presented a theoretical analysis of the linear and non-linear optical properties of realistic quantum wires. Our approach is based on a numerical solution of the semiconductor Bloch equations describing the multisubband 1D system. We have applied such approach to typical T- and V-shaped structures, whose parameters reflect the current state-of-the-art in the quantum-wire fabrication.

Our results allow us to reconsider the perspectives of quantum-wire physics and technology. In particular, comparing the non-linear absorption spectra of Fig. 3(a) and (b), we see that the broad gain region in (b) is mainly ascribed to the multisubband nature or, more precisely, to the small intersubband splitting compared to the single-subband gain range in (a). This confirms that, in order to obtain sharp gain profiles, one of the basic steps in quantum-wire technology is to produce structures with increased subband splitting. However, the disorder-induced inhomogeneous broadening, not considered here, is known to increase significantly the spectral broadening [8] and this effect is expected to increase with increasing subband splitting. Therefore, extremely-high-quality structures (e.g. singlemonolayer control) seem to be the only possible candidates for successful quantum-wire applications. 


\section{ACKNOWLEDGMENTS}

We thank Roberto Cingolani and Guido Goldoni for stimulating and fruitful discussions.

This work was supported in part by the EC Commission through the Network "Ultrafast". 


\section{REFERENCES}

[1] See e.g. J. Shah, Ultrafast Spectroscopy of Semiconductors and Semiconductor Nanostructures (Springer, Berlin, 1996); H. Haug and S.W. Koch, Quantum Theory of the Optical and Electronic Properties of Semiconductors, 3rd Edn., World Scientific, Singapore (1994).

[2] For a review see: R. Cingolani and R. Rinaldi, Rivista Nuovo Cimento 16, 1 (1993).

[3] Tetsuo Ogawa and Toshihide Takagahara, Phys. Rev. B43, 14325 (1991); ibid. B44, 8138 (1991).

[4] E. Kapon et al., Phys. Rev. Lett. 63, 430 (1989).

[5] R. Rinaldi et al., Phys. Rev. Lett. 73, 2899 (1994).

[6] L. Pfeiffer et al., Appl. Phys. Lett. 56, 967 (1990); W. Wegscheider et al., Appl. Phys. Lett. 65, 2510 (1994).

[7] T. Someya, H. Akiyama, and H. Sakaki, Phys. Rev. Lett. 76, 2965 (1996).

[8] E. Molinari et al., in Proc. 22nd Internat. Conf. on the Physics of Semiconductors, edited by D.J. Lockwood, World Scientific, Singapore (1994), p. 1707.

[9] F. Rossi and E. Molinari, Phys. Rev. Lett. 76, 3642 (1996); Phys. Rev. B53, 16462 (1996).

[10] F. Rossi, G. Goldoni, and E. Molinari, Phys. Rev. Lett. 78, 3527 (1997).

[11] T. Meier, F. Rossi, P. Thomas, and S.W. Koch, Phys. Rev. Lett. 75, 2558 (1995).

[12] All the spectra were computed assuming a phenomenological energy broadening of 2 meV. This small broadening (as compared to that of realistic wire structures [8]) allows to better identify the role played by electron-hole correlation.

[13] As for the case of 3D and 2D systems (see e.g. R. Cingolani et al., Adv. Phys. 40, 535 
(1991); Phys. Rev. B48, 14331 (1993), and refs. therein), our analysis seems to indicate a moderate red-shift of the band gap. However, in order to provide a detailed analysis of the band-gap renormalization, a more refined screening model (including non-diagonal terms of the dielectric tensor as well as dynamic-screening effects) is required. 


\section{FIGURES}

FIG. 1. Comparison between the absorption spectra obtained by including electron-hole Coulomb correlation (CC model, solid line) or by assuming free carriers (FC model, dashed line) for the case of: (a) the GaAs/AlGaAs V-wires of Ref. [5]; (b) the GaAs/AlAs T-shaped wire of Ref. [7](see text.) All spectra were computed assuming an energy broadening of $2 \mathrm{meV}$.

FIG. 2. (a) Oscillator-strength ratio between CC and FC spectra (solid line) and electron-hole DOS (dashed line) as a function of the excess energy. (b) Electron-hole correlation function $g(z)$ vs. relative distance $z$ for three different values of the excess energy, identified by the corresponding symbols in (a). Note that $g(z=0)$ gives directly the oscillator strength for the corresponding excess energy (see text).

FIG. 3. Non-linear absorption spectra of the V-shaped wire of Fig. 1(a) at room temperature for different carrier densities: $\mathrm{A}=10^{4} \mathrm{~cm}^{-1} ; \mathrm{B}=5 * 10^{5} \mathrm{~cm}^{-1} ; \mathrm{C}=10^{6} \mathrm{~cm}^{-1} ; \mathrm{D}=4 * 10^{6} \mathrm{~cm}^{-1}$; $\mathrm{E}=2 * 10^{7} \mathrm{~cm}^{-1}$. (a) single-subband case; (b) realistic 12-subband case. For the highest densities [case D in (a) and E in (b)] the corresponding FC result is also shown (line marked with diamonds). For a better comparison of the lineshapes, the FC band edge has been red-shifted to align with the corresponding CC one. 

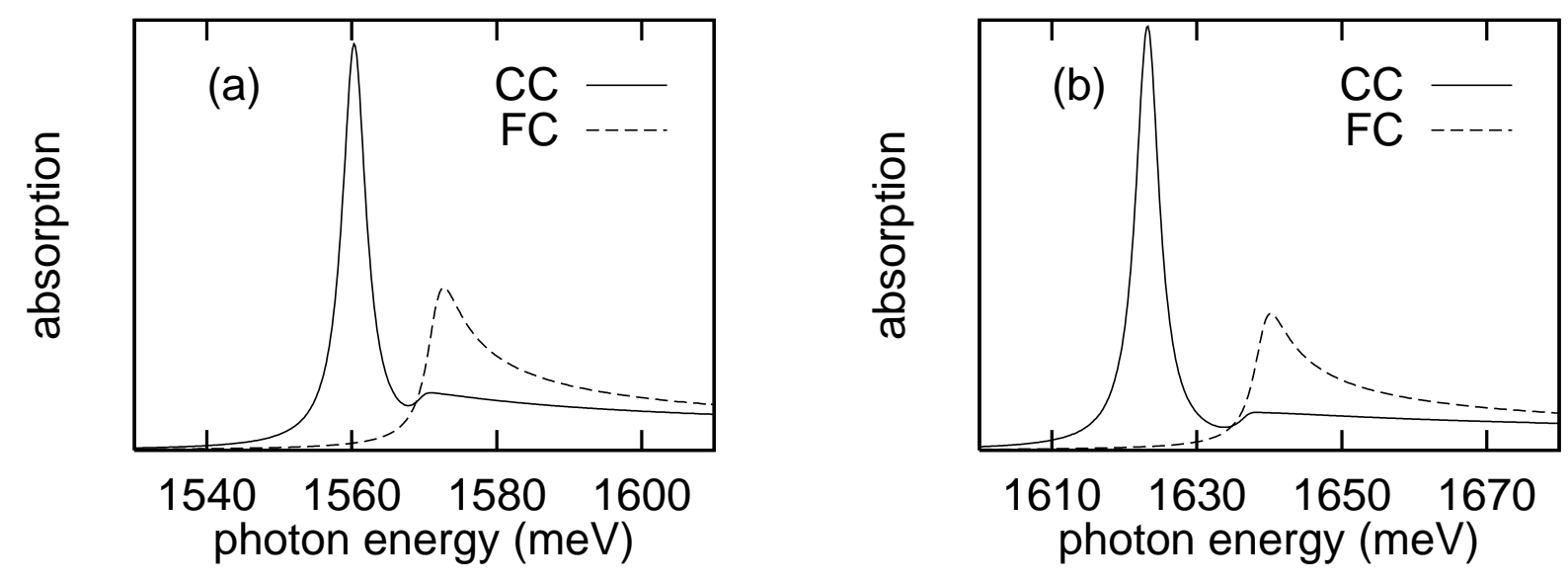

F. Rossi and E. Molinari: Fig. 1 

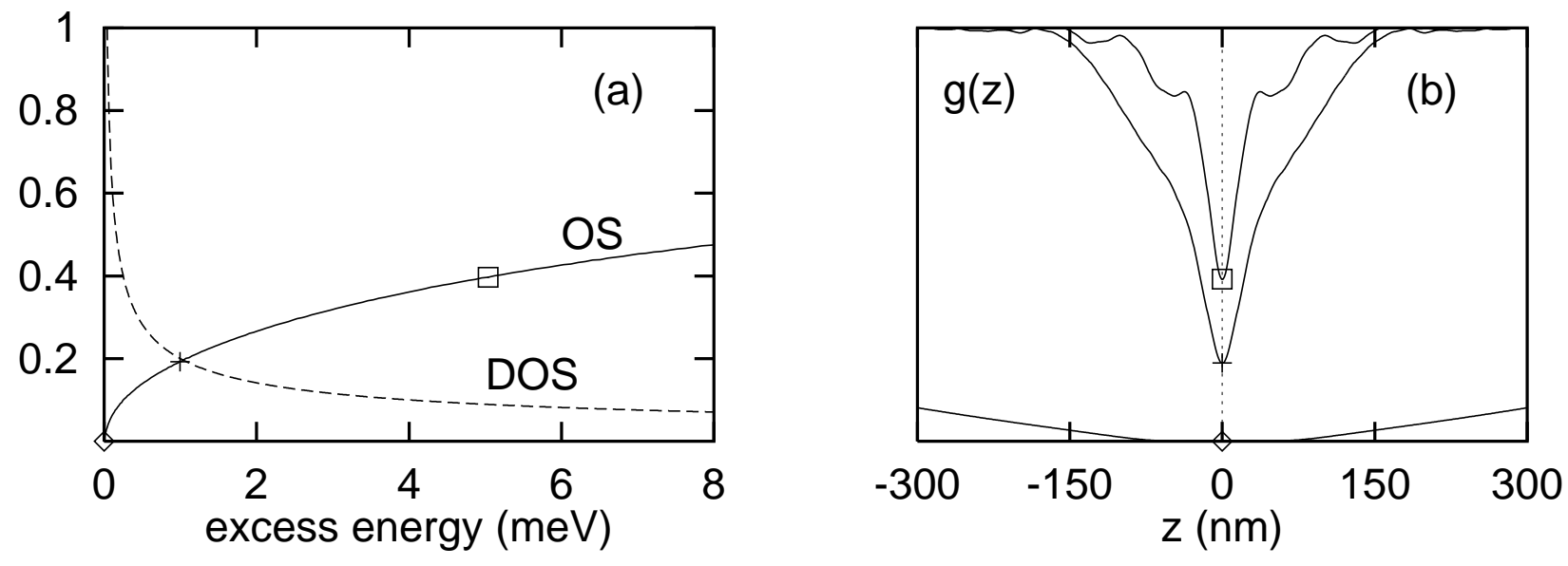

F. Rossi and E. Molinari: Fig. 2 

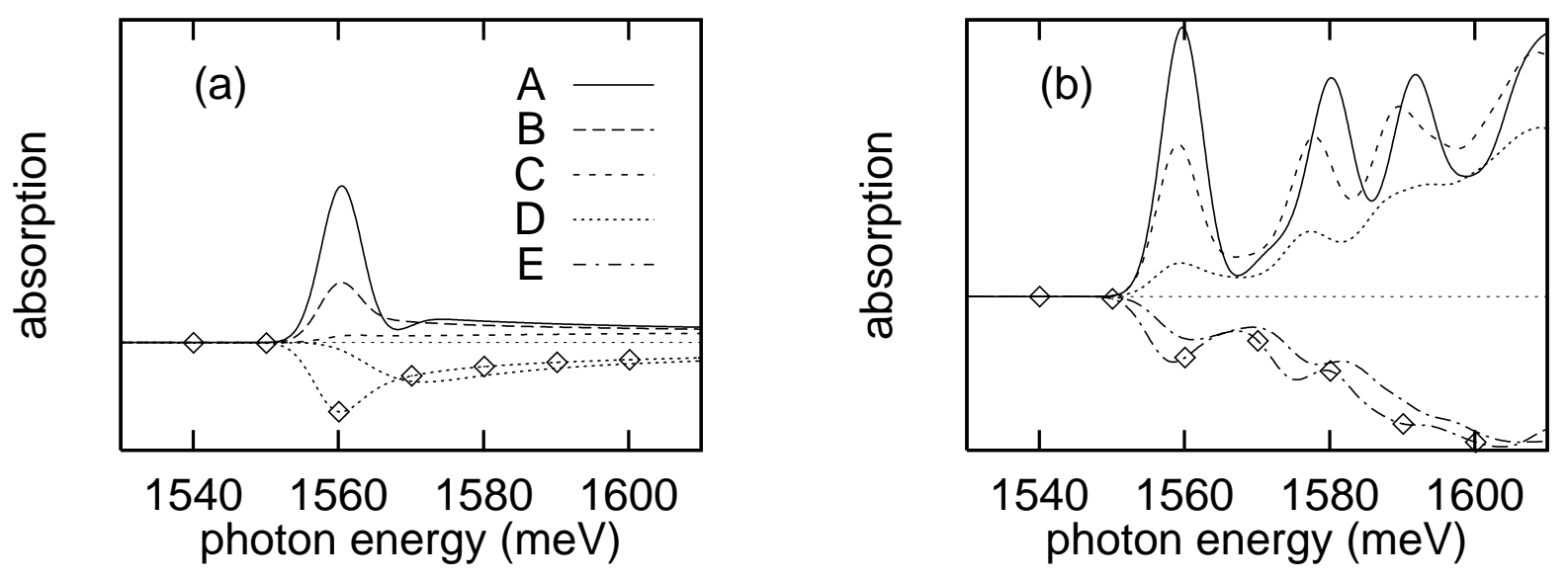

F. Rossi and E. Molinari: Fig. 3 